\section{Factors influencing the renal arterial Doppler waveform: a simulation study using an electrical circuit model (secondary publication)}

\author{
Chang Kyu Sung ${ }^{1}$, Bong Soo Han², Seung Hyup Kim³ \\ 'Department of Radiology, SMG-SNU Boramae Medical Center, Seoul National University \\ College of Medicine, Seoul; ${ }^{2}$ Department of Radiological Science, College of Health Science, \\ Yonsei University, Wonju; ${ }^{3}$ Department of Radiology, Institute of Radiation Medicine, Seoul \\ National University Hospital, Seoul National University College of Medicine, Seoul, Korea
}

Purpose: The goal of this study was to evaluate the effect of vascular compliance, resistance, and pulse rate on the resistive index (RI) by using an electrical circuit model to simulate renal blood flow.

Methods: In order to analyze the renal arterial Doppler waveform, we modeled the renal bloodflow circuit with an equivalent simple electrical circuit containing resistance, inductance, and capacitance. The relationships among the impedance, resistance, and compliance of the circuit were derived from well-known equations, including Kirchhoff's current law for alternating current circuits. Simulated velocity-time profiles for pulsatile flow were generated using Mathematica (Wolfram Research) and the influence of resistance, compliance, and pulse rate on waveforms and the RI was evaluated.

Results: Resistance and compliance were found to alter the waveforms independently. The impedance of the circuit increased with increasing proximal compliance, proximal resistance, and distal resistance. The impedance decreased with increasing distal compliance. The RI of the circuit decreased with increasing proximal compliance and resistance. The RI increased with increasing distal compliance and resistance. No positive correlation between impedance and the RI was found. Pulse rate was found to be an extrinsic factor that also influenced the RI.

Conclusion: This simulation study using an electrical circuit model led to a better understanding of the renal arterial Doppler waveform and the RI, which may be useful for interpreting Doppler findings in various clinical settings.

Keywords: Renal artery; Ultrasonography, Doppler; Computer simulation

\section{Introduction}

The resistive index (RI), pulsatility index, systolic/diastolic ratio, peak systolic velocity (PSV),

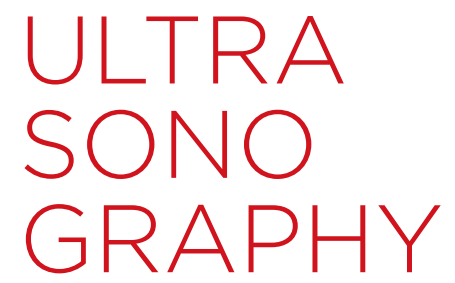

ORIGINAL ARTICLE

http://dx.doi.org/10.14366/usg.15056 pISSN: 2288-5919 • elSSN: 2288-5943 Ultrasonography 2016:35:69-77

Received: September 15, 2015

Revised: September 21, 2015

Accepted: September 21, 2015

Correspondence to:

Seung Hyup Kim, MD, Department of Radiology, Seoul National University Hospital, 101 Daehak-ro, Jongno-gu, Seoul 03080, Korea

Tel. $+82-2-2072-2510$

Fax. +82-2-743-6385

E-mail: kimsh@radcom.snu.ac.kr

This article is an English version of "Evaluation of factors affecting the renal Doppler waveform with the use of an electrical circuit model [Korean]," published in the Journal of Korean Society of Ultrasound in Medicine in June 2009.

This is an Open Access article distributed under the terms of the Creative Commons Attribution NonCommercial License (http://creativecommons.org/ licenses/by-nc/3.0/) which permits unrestricted noncommercial use, distribution, and reproduction in any medium, provided the original work is properly cited.

Copyright (C) 2016 Korean Society of Ultrasound in Medicine (KSUM)

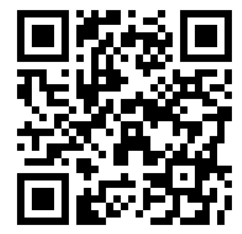

Please cite the original article as: Sung CK, Han BS, Kim SH. Evaluation of factors affecting the renal Doppler waveform with the use of an electrical circuit model. J Korean Soc Ultrasound Med. 2009 Jun;28(2): 75-82. 
acceleration time, acceleration index, and other parameters are used to quantitatively analyze the characteristics of Doppler waveforms of renal blood flow. Of these parameters, the RI is most commonly used clinically. Since consistent RI values are obtained regardless of the Doppler incident angle, the RI is suitable for examining the renal artery with an irregular direction of blood flow. The RI has been recognized as a useful parameter in several renal disorders, including acute tubular necrosis, obstructive or medical nephropathy, hepatorenal syndrome, renal cell carcinoma in patients with end stage renal disease, and others [1-7]. The RI was previously regarded as a specific indicator for evaluating the hemodynamics of transplanted kidneys. Many reports have found the RI to be a valuable Doppler parameter for the assessment of renal transplant dysfunction [8-11]. However, several studies have found that the RI lacks specificity in the evaluation of renal transplants [12-14]. Many researchers have agreed that RI values from Doppler examinations obtained at different times may provide useful information for monitoring the progress of an allograft, evaluating therapeutic efficacy, and detecting subclinical atherosclerotic damage in the cardiovascular system of transplant recipients $[10,12,15]$. It seems that the interpretation of the $\mathrm{RI}$ in renal Doppler studies is more complex than originally thought. Most previous studies hypothesized that the RI reflects changes in renal arterial resistance that occur over the course of renal disease, and some studies considered the $\mathrm{RI}$ and renal arterial resistance to be equivalent concepts [16-18]. However, despite the verification of its clinical utility, an insufficient understanding exists regarding changes in RI values, and basic research explaining the fundamental characteristics of $\mathrm{Rl}$ is lacking.

Many animal experiments and studies using a blood flow phantom have proposed that vascular RI not only reflects resistance to blood flow, but also is affected by several other factors, including vascular compliance [19-21]. Since blood flow circuits are in many ways similar to electrical circuits, the components of a blood flow circuit (vascular resistance, inductance, and compliance) can be considered equivalent to the components of an electrical circuit (resistance, inductance, and capacitance) [22]. Using this analogy, useful information can be expressed by modeling blood flow in the cardiovascular system as an electrical circuit, using equations for electrical circuits. Simulations of this type allow a range of variables to be defined precisely, in contrast to animal tests or studies using other techniques to model blood flow. Through this process, a detailed and comprehensive analysis of the RI is possible, incorporating associations between the RI and variables affecting the RI, as well as the interactions among these variables. This study aimed to investigate the effect of factors such as vascular compliance and resistance on the RI based on an electrical circuit simulation model.

\section{Materials and Methods}

We used a model of the renal blood flow circuit containing vascular resistance, inductance, and compliance, equivalent to an electrical circuit containing resistance, inductance, and capacitance [22]. This electrical circuit model simulated renal blood flow. We simulated a resistor-capacitor circuit, simplifying the circuit to have resistance and capacitance only, based on the hypothesis that a blood flow circuit is not influenced by inductance, which is a property of a conductor (typically a conducting coil) in which electromotive force is produced by electromagnetic induction (Fig. 1). Based on this model and by using well-known equations for electrical circuits, blood flow velocity $(f)$ at the sampling site of Doppler sonography can be expressed as the inverse of the total impedance, which is the vector sum of electrical resistance.

$f / P_{\text {in }}=1 / Z \quad\left(f\right.$, flow velocity; $P_{\text {in, }}$ input pressure; $Z$, impedance $)$

According to Kirchhoff's laws for an alternating current circuit, the following three equations are obtained [23].

$$
\begin{aligned}
& P_{\text {in }}-P_{\text {out }}=f R+f_{1} R_{1}+f_{2} R_{2} \\
& C_{1} d\left(P_{\text {in }}-R_{1} f_{1}\right) / d t=f_{1}-f \\
& C_{2} d\left(R_{2} f_{2}+P_{\text {out }}\right) / d t=f-f_{2}
\end{aligned}
$$

When these equations are solved, impedance can be expressed as a function of resistance and compliance as follows:

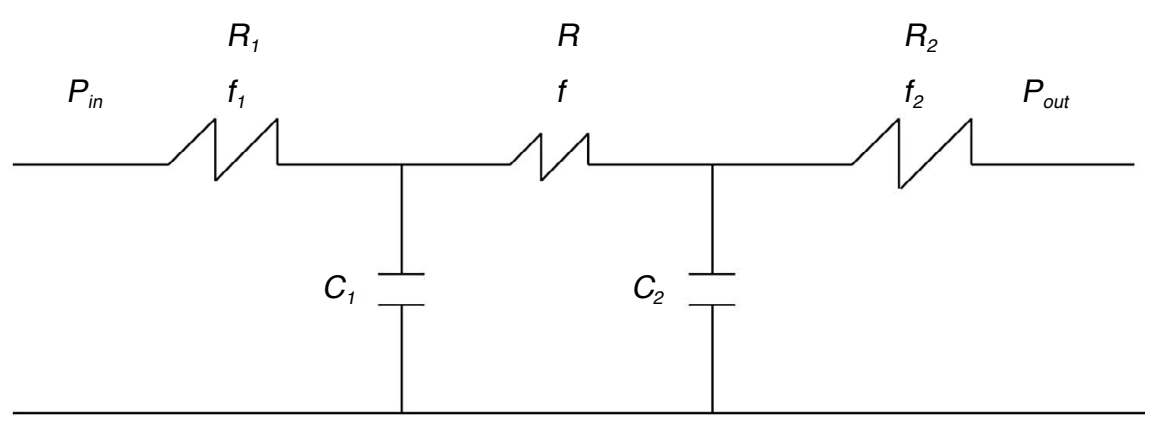

Fig. 1. The simulated electrical circuit model of the renal blood-flow circuit. $R_{1}$, proximal resistance; $R_{2}$, distal resistance; $f_{1,}$ proximal flow rate; $f_{2}$, distal flow rate; $C_{1}$, proximal compliance; $C_{2}$, distal compliance; $P_{\text {in, }}$ input pressure; $P_{\text {out }}$ output pressure; $R$ and $f$, the resistance and flow rate at the measurement point, respectively. 


$$
Z=\frac{\sqrt{\left[R+R_{1}+R_{2}+\omega^{2} C_{2} R_{2}\left(C_{1} R R_{1}+C_{1} R_{1} R_{2}-C_{2} R_{2}^{2}\right)\right]^{2}+\omega^{2}\left[\left(R+R_{2_{1}}\right)\left(C_{1} R_{1}-C_{2} R_{2}\right)-C_{2} R_{2}\left(R_{1}+R_{2}\right)\right]^{2}}}{1+\omega^{2} C_{2}^{2} R_{2}^{2}}
$$

In the above equation, $\omega$ denotes the frequency of $P_{i n}$ and $R$ is the resistance at the measuring point of $f$. $R$ is expected to be a very small value. Impedance was graphed along with the resistance and compliance of the proximal and distal areas by using the above four equations in Mathematica (Wolfram Research, Champaign, IL, USA). Changes in impedance were simulated in response to changes of the variables that affect blood flow.

Arterial flow results in waveforms showing the difference between the systolic and diastolic pressures. In order to simulate renal arterial blood flow in an in vivo cardiac cycle, the pulse rate was set at $1 \mathrm{~Hz}$ (60 beats per minute) with a 50\% systolic and a $50 \%$ diastolic component, the maximum pressure was set at 50 $\mathrm{mm} \mathrm{Hg}$, and the diastolic pressure was set at $0 \mathrm{~mm} \mathrm{Hg}$ (Fig. 2). Several types of waveforms reflecting changes in the proximal and distal values of resistance and compliance were drawn using Mathematica. Subsequently, the influence of these factors on flow velocity and the RI was evaluated according to changes in each variable. The RI was obtained using the formula [(PSV-minimum diastolic velocity [MDV])/PSV]. The basic standard waveforms are presented as a thick line when the regular pulse in Fig. 2 is provided with proximal compliance $\left(C_{1}\right)$ set to 1.3 , distal compliance $\left(C_{2}\right)$ set to 0.9 , proximal resistance $\left(R_{1}\right)$ set to 1.0 , and distal resistance $\left(R_{2}\right)$ set to 0.8 . These are arbitrarily chosen values that result in a waveform similar to renal arterial Doppler waveforms. In order to draw Doppler waveforms representing the influence of each

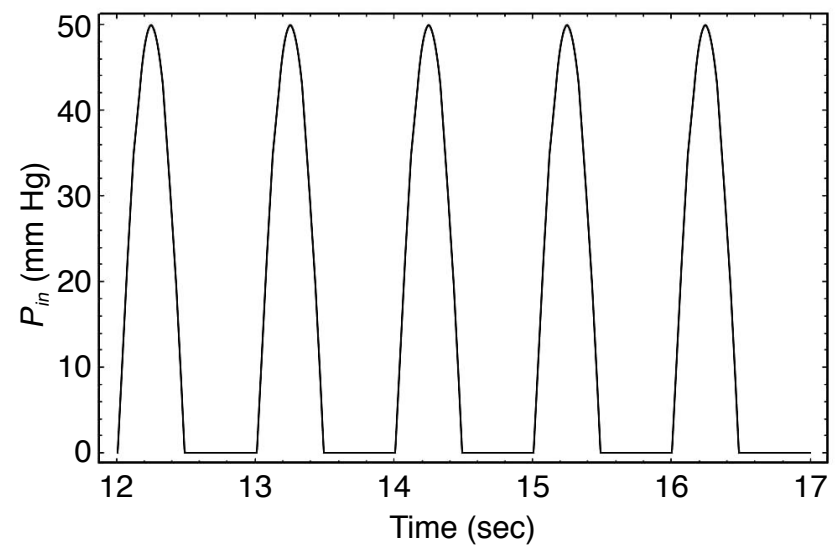

Fig. 2. Diagram of a pulse wave. The pulse rate was $1 \mathrm{~Hz}(60$ beats per minute) with a $50 \%$ systolic phase component and a $50 \%$ diastolic phase component. The systolic pressure curves show a sinusoidal curve with a maximum pressure of $50 \mathrm{~mm} \mathrm{Hg}$ and a diastolic pressure of $0 \mathrm{~mm} \mathrm{Hg}$. $P_{i n,}$ input pressure. component, the waveforms resulting from a threefold increase of each variable were presented as a thin line and compared with the basic waveforms. Nevertheless, non-uniformity may occur depending on the degree of hardening of the arteries, since our model implies that the proximal and distal compliance values are changed at the same ratio as arteriosclerotic changes progress evenly throughout the arteries. Basic waveforms were compared with the waveforms created when the proximal and distal compliances were increased threefold or decreased by one third in order to evaluate the effects of atherosclerotic changes. In order to evaluate the impact of pulse rate on the RI independently, differences in the basic waveforms were evaluated by altering the pulse rate and maintaining a consistent magnitude and shape of the pressure ripple. Since a reduced blood flow quantity is associated with a decreased heart rate, the pressure ripple and resistance must be increased in order to maintain a consistent quantity of blood flow. Therefore, the effect of changes in the pulse rate on the RI was also examined by altering the magnitude of the pressure ripple.

\section{Results}

We simulated a simple electrical circuit with resistance and capacitance only and graphed the changes in impedance in response to changes in different variables using well-known equations for electrical circuits (Fig. 3). Based on these graphs, the impedance that influences blood flow in the Doppler sampling site, increased with increasing proximal compliance, proximal resistance, and distal resistance, and decreased with increasing distal compliance (Table 1).

The effects of changes of the proximal and distal resistance and compliance values on flow velocity and the RI were evaluated using waveforms created in Mathematica. When proximal compliance was increased, the PSV decreased and the MDV increased, thereby decreasing the RI (Fig. 4A). When distal compliance was increased, the PSV increased and the MDV decreased, thereby increasing the

Table 1. The relationship between variables and impedance/the resistive index

\begin{tabular}{lcc}
\hline & Impedance & Resistive index \\
\hline Proximal compliance $\left(C_{1}\right) \uparrow$ & $\uparrow$ & $\downarrow$ \\
Proximal resistance $\left(R_{1}\right) \uparrow$ & $\uparrow$ & $\downarrow$ \\
Distal compliance $\left(C_{2}\right) \uparrow$ & $\downarrow$ & $\uparrow$ \\
Distal resistance $\left(R_{2}\right) \uparrow$ & $\uparrow$ & $\uparrow$ \\
\hline
\end{tabular}

$\uparrow$, an increase; $\downarrow$, a decrease. 
RI (Fig. 4B). When proximal resistance increased, the PSV decreased and the MDV remained unchanged, thus decreasing the RI (Fig. 4C). Although the PSV decreased as the distal resistance increased, the RI increased as the MDV decreased to a greater extent (Fig. 4D). When the proximal and distal compliance values were increased threefold or decreased by one third, their waveforms were nearly the same as the waveforms drawn before the increase or decrease (Fig. 5). Uniform changes of compliance throughout the arteries had almost no influence on the RI.

When the pulse rate was altered from one beat per second to one beat per two seconds and one beat per four seconds, the RI increased, since the degree of decrease was greater in the MDV than in the PSV (Fig. 6A). The slower the pulse rate, the smaller the blood

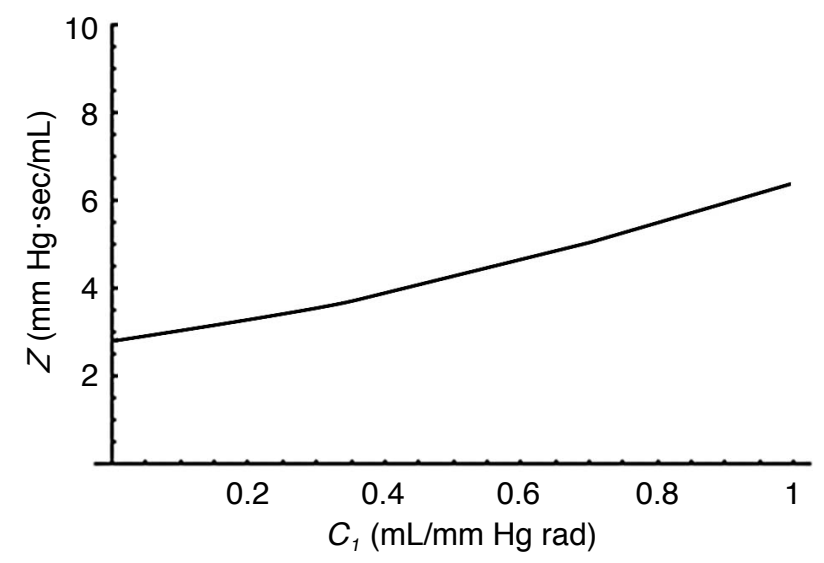

A

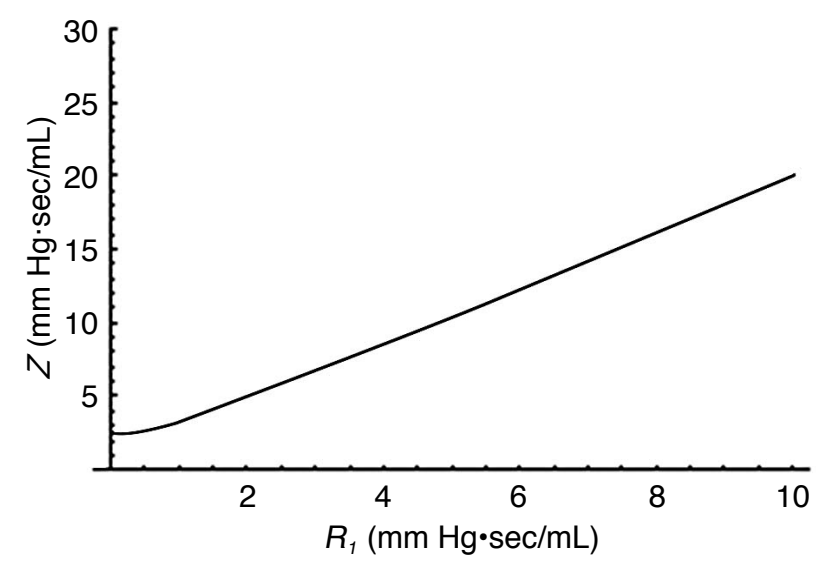

C

Fig. 3. Graphs of the relationships of impedance with proximal compliance, distal compliance, proximal resistance, and distal resistance. The Y-axis represents the impedance. A. With increasing proximal compliance, the impedance increased in a relatively linear manner. B. With increasing distal compliance, the impedance decreased like a graph of the reciprocal function. C. With increasing proximal resistance, the impedance increased with in a relatively linear manner. D. With increasing distal resistance, the impedance increased and then reached a plateau. $Z$, impedance; $C_{1}$, proximal compliance; $C_{2}$ distal compliance; $R_{1}$, proximal resistance; $R_{2}$, distal resistance.

flow amount. Despite changes in the pulse rate, the magnitude of the pressure ripple can be altered to maintain a consistent blood flow amount. Under these circumstances, the RI also increased as the pulse rate declined (Fig. 6B). However, changes in only the magnitude of the pressure ripple had no influence on the RI (Fig. 6C).

\section{Discussion}

The circulatory system in the body is in many ways similar to an electrical circuit. For this reason, vascular resistance, inductance, and compliance can be considered equivalent to the corresponding concepts in electrical circuits. Impedance, which is the sum of

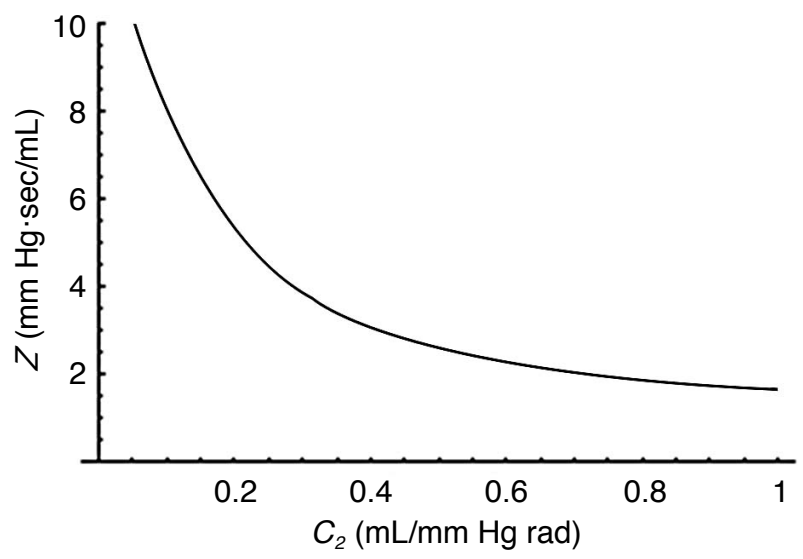

B

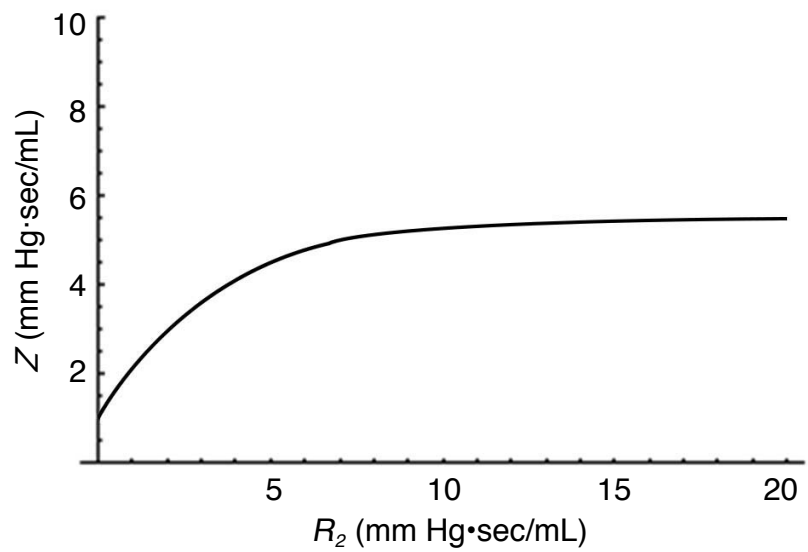

D 
electrical resistance in alternating current circuits, is commonly used to understand dynamic changes in vascular flow. Impedance represented as the ratio of voltage to the current wave in an alternating circuit can replace the notion of resistance, since vascular flow with regular heartbeats has similarities to alternating current electricity. Although the dictionary definition of impedance is very similar to that of resistance, a significant difference is that impedance is applied to an alternating circuit with a given frequency. Thus, impedance includes the concepts of inductance and capacitance which involve resistors in conditions where frequency is a relevant parameter, as well as encompassing the concept of resistance itself, for which frequency is irrelevant. When an electric current flows through a coil of wire, temporarily stored energy (magnetic field energy in the case of inductance or electric field energy in the case of capacitance) seems to be consumed. Even though energy is actually used up in some cases, the accumulated energy is typically recycled in an alternating current circuit. Likewise, impedance differs according to the frequency caused by alternating

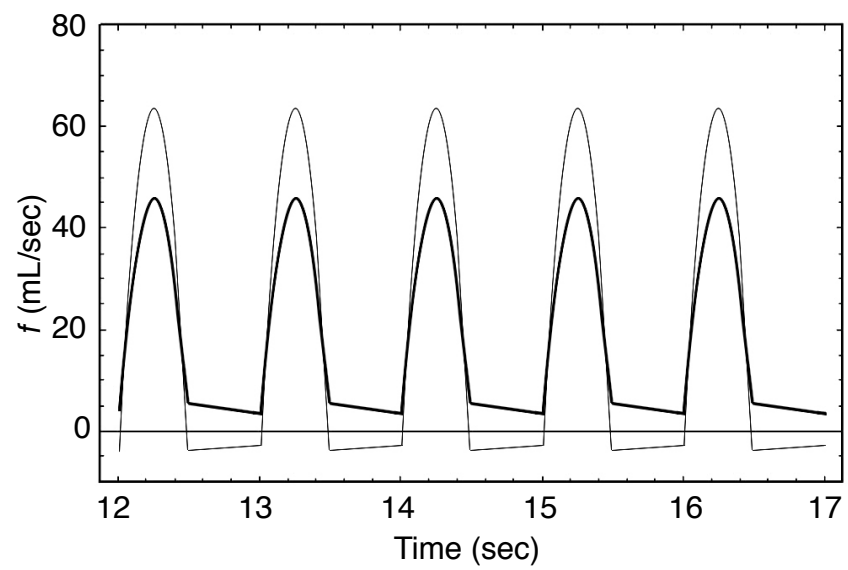

B

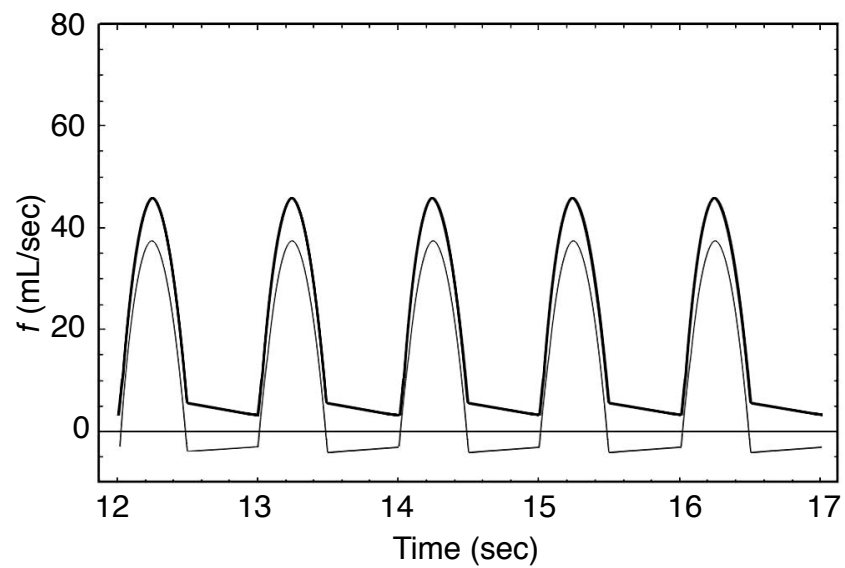

D

Fig. 4. The effects of resistance and compliance on the simulated flow waveforms.

A. A thick line indicates the waveform at the following settings: $C_{1}, 1.3 ; C_{2}, 0.9 ; R_{1}, 1.0$; and $R_{2}, 0.8$. A thin line indicates the waveform when the proximal compliance $\left(C_{1}\right)$ was increased threefold (3.9). The peak systolic velocity (PSV) decreased and the minimum diastolic velocity (MDV) increased. Therefore, the resistive index (RI) decreased when the proximal compliance was increased. $B$. The thin line indicates the waveform when the distal compliance $\left(C_{2}\right)$ was increased threefold (2.7). The PSV increased and the MDV decreased. Therefore, the RI increased when distal compliance was increased. $C$. The thin line indicates the waveform when the proximal resistance $\left(R_{1}\right)$ was increased threefold (3.0). The PSV decreased and the MDV did not change. Therefore, the RI decreased when the proximal resistance was increased. D. Thin line indicates the waveform when the distal resistance $\left(R_{2}\right)$ was increased threefold (2.4). The PSV decreased and the MDV was inverted; therefore, the RI increased when the distal resistance is increased. $f$, flow velocity; $C_{1}$, proximal compliance; $C_{2}$, distal compliance; $R_{1}$, proximal resistance; $R_{2}$, distal resistance. 

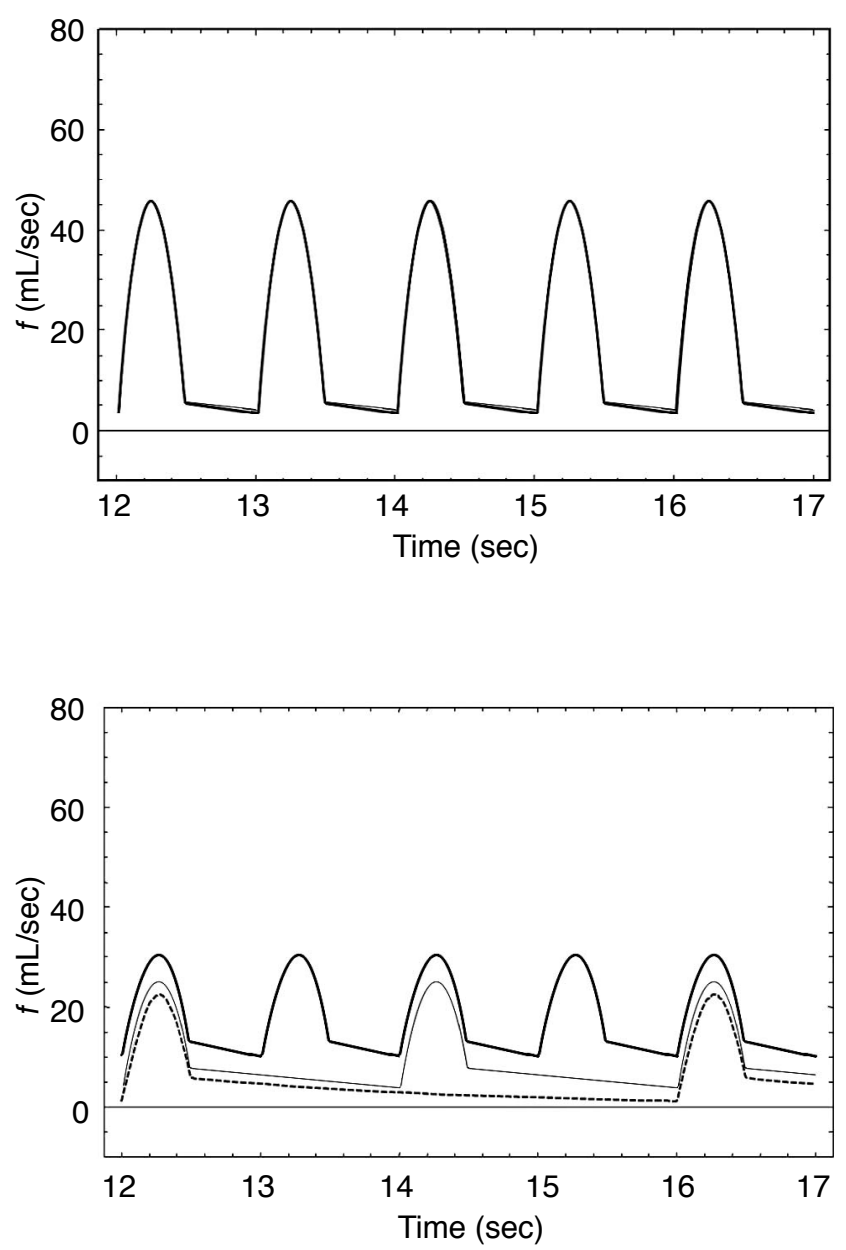

A

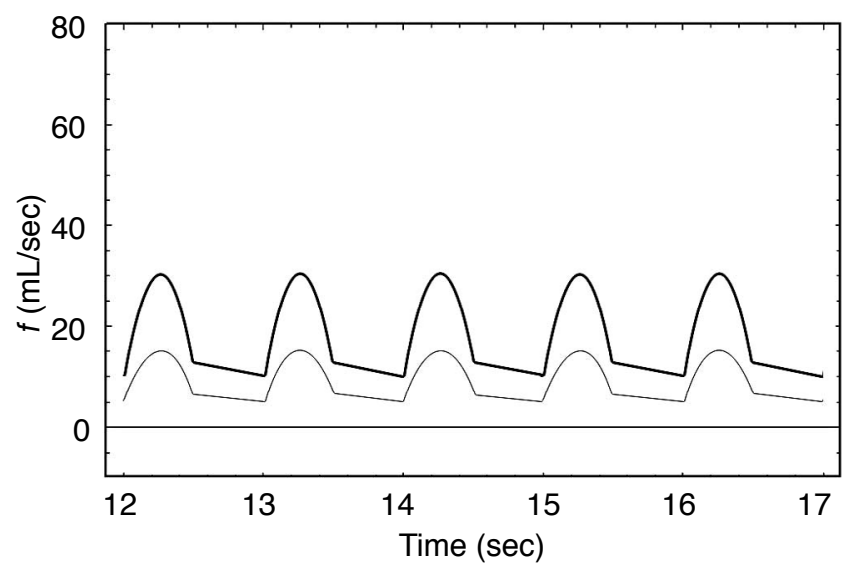

C

decreased and the pulse pressure simultaneously increased, the PSV increased and the MDV slightly decreased, meaning that the RI was increased. $C$. The thick line indicates the waveform at a pulse rate of 60 beats per minute; a pulse pressure of $100 ; C_{1}, 3.9 ; C_{2}, 0.9 ; R_{1}, 1.0$; and $R_{2}, 0.8$. The thin line indicates the waveform at a pulse pressure of 50 with other variables held constant. As the pulse pressure was decreased, the PSV decreased and the MDV decreased to the same degree, meaning that the RI did not change. $f$, flow velocity; $R_{1}$, proximal resistance; $R_{2}$, distal resistance; $C_{1}$, proximal compliance; $C_{2}$, distal compliance.
Fig. 5. The effects of simultaneously changing proximal and distal compliance on the simulated flow waveform. The waveform is almost not changed when the proximal compliance was increased with a simultaneous increase of distal compliance of the same magnitude (thick line: $C_{1}, 1.3 ; C_{2}, 0.9 ; R_{1}, 1.0 ; R_{2}, 0.8$; thin line: $C_{1}, 3.9 ; C_{2}, 2.7$; $\left.R_{1}, 1.0 ; R_{2}, 0.8\right)$. f, flow velocity; $C_{1}$, proximal compliance; $C_{2}$, distal compliance; $R_{1}$, proximal resistance; $R_{2}$, distal resistance.

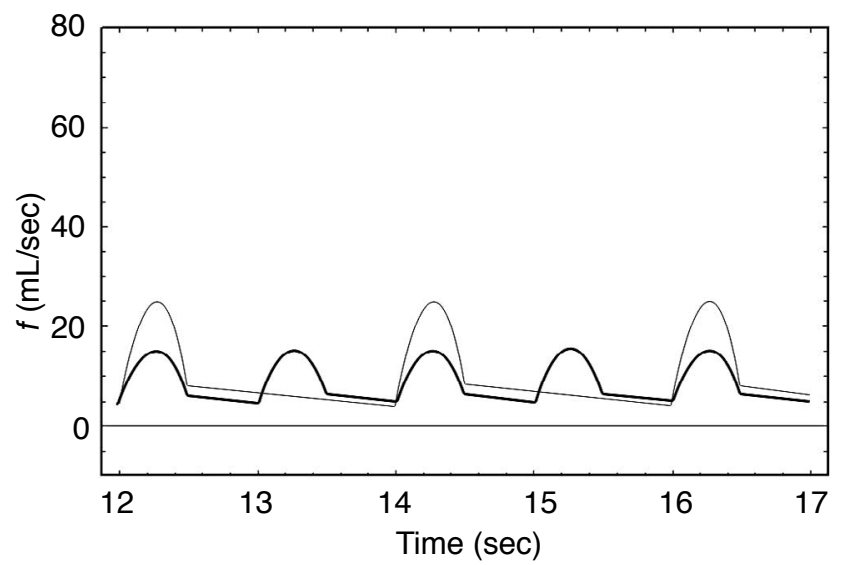

B

Fig. 6. The effects of pulse rate and pulse pressure on the simulated flow waveforms.

A. The thick line indicates the waveform at pulse rate of 60 beats per minute; a pulse pressure of $100 ; C_{1}, 3.9 ; C_{2}, 0.9 ; R_{1}, 1.0$; and $R 2,0.8$. The thin line indicates the waveform at a pulse rate of 30 beats per minute and the dashed line indicates the waveform at a pulse rate of 15 beats per minute. The other variables are same as the waveform depicted with the thick line. The peak systolic velocity (PSV) slightly decreased and the minimum diastolic velocity (MDV) decreased to a greater extent, meaning that the resistive index (RI) increased when the pulse rate was decreased. B. The thick line indicates the waveform at a pulse rate of 60 beats per minute; a pulse pressure of $50 ; C_{1}, 3.9 ; C_{2}, 0.9 ; R_{1}, 1.0$; and $R_{2}, 0.8$. The thin line indicates the waveform at a pulse rate of 30 beats per minute and a pulse pressure of 100 . The other variables are the same as in the waveform depicted with the thick line. As the pulse rate was 
resistors and load. Thus, impedance, which embraces the concepts of accumulation and load in addition to power consumption, is a more complex version of resistance that is applied to circuits that involve frequency. Unlike electrical resistance in a direct current circuit, it cannot be concluded that resistance and impedance are interchangeable in an alternating circuit current.

Early studies on changes in Doppler waveforms and the RI investigated the relationship between the RI and resistance by focusing on its relationship with distal resistance [16-18]. However, some studies have addressed the roles of both vascular resistance and compliance $[20,21]$. This simulation study, which was conducted using an electrical circuit model, was able to determine that changes in compliance alter flow waveforms and the RI. Impedance and the $\mathrm{RI}$ increased inversely in response to changes in proximal or distal compliance. Bude and Rubin [20] performed a study using a blood flow model and argued that the impedance index is a better metric because flow Doppler waveforms are affected by resistance and compliance. The present study showed that changes in impedance and the $\mathrm{RI}$ in response to changes in distal resistance had the same directionality. In contrast, impedance changed in the opposite direction to the RI when the distal compliance, proximal compliance, or proximal resistance was changed. Therefore, expressing the impedance index in terms of the RI is inaccurate, since the directionality of change in impedance and the $\mathrm{RI}$ in response to the change of variables involved in blood flow is inconsistent.

When arterial elasticity decreases due to vascular aging, compliance is reduced. However, when the magnitude of the pressure ripple and the quantity of blood flow are the same, the total resistance remains unchanged. When the proximal and distal compliance values were changed at the same ratio, the resulting waveforms were nearly the same as the waveforms drawn before the change. When compliance is reduced at the same ratio as vascular hardening that progresses evenly throughout the arteries, these changes in compliance have almost no effect on the RI. However, evaluating only the effect of arteriosclerosis on the RI is difficult in the actual clinical setting, because it is not always possible to exclude the influence of other variables, such as resistance, and because hardening can occur unevenly in the arterial wall. For these reasons, the effect of arteriosclerosis on the $\mathrm{RI}$ is difficult to predict. Although Shimizu et al. [24] and Ohta et al. [25] have found that the degree of progression of arteriosclerosis is correlated with an increase in the renal arterial $\mathrm{RI}$, this outcome does not mean that a decrease in vascular compliance induces an increase in the RI. Since hemodynamic changes in renal arteries and histological changes in the renal parenchyma are commonly associated with each other, changes in the RI due to arteriosclerosis or hypertension are not only affected by compliance but also changes in other factors, such as resistance caused by renal parenchymal damage.

In addition to resistance and compliance, Doppler waveforms are also influenced by cardiac function, the anatomical structure of the blood vessels, and other factors. Cardiac functions affecting waveforms include systolic and diastolic pressure, systolic and diastolic time intervals, pulse pressure, cardiac output, and pulse rate. Since these factors are closely associated with each other, it is difficult to analyze them independently. Mostbeck et al. [26] reported a significant correlation between the RI and heart rate. Contrastingly, Kublickas et al. [27] found no association between the $\mathrm{RI}$ and heart rate. The presence of contradicting results may be attributed to the fact that these previous studies involved human subjects. Thus, heart rate was not manipulated to an extent sufficient to generate a change in the RI. It is also possible that the results were affected by other cardiac factors that presumably could not be completely excluded. In this simulation study, we were able to observe the effect of pulse rate by significantly changing the pulse rate and maintaining a consistent cardiac output and pulse pressure. The faster the pulse rate, the shorter the diastolic time compared to the systolic time. When the pulse rate becomes faster, the PSV remains almost the same, while the RI decreases with an increase in the MDV, as systole takes place without a sufficient period of diastole. Since compensatory physiological responses occur to maintain normal blood flow amount and blood pressure as the pulse rate becomes slower, the hidden impact of pulse rate changes must be taken into consideration. In order to maintain a consistent blood flow volume despite changes in heart rate, the magnitude of the pressure ripple may change. In our study, the RI increased in response to decreases in the pulse rate and the $\mathrm{RI}$ increased in response to decrease in the pulse rate with simultaneous increase in magnitude of the pressure ripple. In contrast, when only the magnitude of the pressure ripple was changed with a consistent pulse rate, the RI remained the same. Therefore, unlike pulse pressure, the pulse rate is an extrinsic factor that also was found to influence the RI.

This investigation simulated changes beyond the limitations of what is possible in vivo by evaluating the effect of changes in each component using a simulated electrical circuit model, and assessed the effect of each variable independently by isolating and changing interdependent variables. Taking into consideration the fact that in vivo vascular flow is more likely than electrical current to be affected by many variables, this analysis is limited because only some factors were evaluated. This study determined that impedance and RI were influenced by both proximal and distal resistance and compliance. Nevertheless, a significant difference in the degree of changes in vascular resistance and compliance must be accounted for. While vascular resistance can range from zero to infinity, the degree of 
changes in vascular compliance is small, despite the effects of arterial hardening, interstitial edema in the distal peripheral region, cellular infiltration, and other factors. Thus, it may be anticipated that these factors will have impacts of different levels on Doppler waveforms and on the $\mathrm{RI}$ in vivo.

Summarizing the results of our study, impedance increased with increasing proximal compliance, proximal resistance, and distal resistance. Impedance decreased with increasing distal compliance. The RI of the circuit decreased with increasing proximal compliance and resistance, and increased with increasing distal compliance and resistance. The impedance changed in the same direction as the RI when the distal resistance was changed. However, the impedance changed in the opposite direction as the RI when the distal compliance, proximal compliance, or proximal resistance was changed. Hence, the changes in RI were not concordant with the changes in impedance in some circumstances. In the absence of changes in intrinsic factors, such as compliance or resistance, the pulse rate can influence the RI as an extrinsic factor. In conclusion, this study was able to identify the effect of different variables on Doppler waveforms and the RI using an electrical circuit model, and the findings of our study are anticipated to be useful in interpreting the changes in Doppler flow waveforms in various clinical settings.

ORCID: Chang Kyu Sung: http://orcid.org/0000-0003-4922-5107; Bong Soo Han: http://orcid.org/0000-0001-6614-7185; Seung Hyup Kim: http://orcid.org/00000002-9339-5907

\section{Conflict of Interest}

No potential conflict of interest relevant to this article was reported.

\section{References}

1. Platt JF, Rubin JM, Ellis JH. Acute renal failure: possible role of duplex Doppler US in distinction between acute prerenal failure and acute tubular necrosis. Radiology 1991;179:419-423.

2. Platt JF, Rubin JM, Ellis JH, DiPietro MA. Duplex Doppler US of the kidney: differentiation of obstructive from nonobstructive dilatation. Radiology 1989;171:515-517.

3. Platt JF, Ellis JH, Rubin JM, DiPietro MA, Sedman AB. Intrarenal arterial Doppler sonography in patients with nonobstructive renal disease: correlation of resistive index with biopsy findings. AJR Am J Roentgenol 1990;154:1223-1227.

4. Kim SH, Kim WH, Choi BI, Kim CW. Duplex Doppler US in patients with medical renal disease: resistive index vs serum creatinine level. Clin Radiol 1992;45:85-87.

5. Colli A, Cocciolo M, Riva C, Martinez E. Abnormal renovascular impedance in patients with hepatic cirrhosis: detection with duplex US. Radiology 1993;187:561-563.
6. Boddi M, Cecioni I, Poggesi L, Fiorentino F, Olianti K, Berardino S, et al. Renal resistive index early detects chronic tubulointerstitial nephropathy in normo- and hypertensive patients. Am J Nephrol 2006;26:16-21.

7. Kim SY, Woo S, Hwang SI, Moon MH, Sung CK, Lee HJ, et al. Usefulness of resistive index on spectral Doppler ultrasonography in the detection of renal cell carcinoma in patients with end-stage renal disease. Ultrasonography 2014;33:136-142.

8. Radermacher J, Mengel M, Ellis S, Stuht S, Hiss M, Schwarz A, et al. The renal arterial resistance index and renal allograft survival. $N$ Engl J Med 2003;349:115-124.

9. Khosroshahi HT, Tarzamni M, Oskuii RA. Doppler ultrasonography before and 6 to 12 months after kidney transplantation. Transplant Proc 2005;37:2976-2981.

10. Krumme B. Renal Doppler sonography: update in clinical nephrology. Nephron Clin Pract 2006;103:c24-c28.

11. Nezami N, Tarzamni MK, Argani H, Nourifar M. Doppler ultrasonographic indexes in kidney transplant recipients: its relationship with kidney function. Iran J Kidney Dis 2007;1:82-87.

12. Heine GH, Gerhart MK, Ulrich C, Kohler H, Girndt M. Renal Doppler resistance indices are associated with systemic atherosclerosis in kidney transplant recipients. Kidney Int 2005;68:878-885.

13. Heine GH, Girndt M, Sester U, Kohler H. No rise in renal Doppler resistance indices at peak serum levels of cyclosporin $A$ in stable kidney transplant patients. Nephrol Dial Transplant 2003;18:16391643.

14. Chiang YJ, Chu SH, Chuang CK, Chen HW, Chou CC, Chen Y, et al. Resistive index cannot predict transplant kidney function. Transplant Proc 2003;35:94-95.

15. Gao J, Rubin JM, Xiang DY, He W, Auh YH, Wang J, et al. Doppler parameters in renal transplant dysfunction: correlations with histopathologic changes. J Ultrasound Med 2011;30:169-175.

16. Norris CS, Barnes RW. Renal artery flow velocity analysis: a sensitive measure of experimental and clinical renovascular resistance. J Surg Res 1984;36:230-236.

17. Spencer JA, Giussani DA, Moore PJ, Hanson MA. In vitro validation of Doppler indices using blood and water. J Ultrasound Med 1991;10:305-308.

18. Halpern EJ, Merton DA, Forsberg F. Effect of distal resistance on Doppler US flow patterns. Radiology 1998;206:761-766.

19. Tublin ME, Tessler FN, Murphy ME. Correlation between renal vascular resistance, pulse pressure, and the resistive index in isolated perfused rabbit kidneys. Radiology 1999;213:258-264.

20. Bude RO, Rubin JM. Relationship between the resistive index and vascular compliance and resistance. Radiology 1999;211:411-417.

21. Halpern EJ, Deane CR, Needleman L, Merton DA, East SA. Normal renal artery spectral Doppler waveform: a closer look. Radiology 1995; 196:667-673.

22. Choi BC, Kim CH, Nam KG, Jung DK, Kim YJ, Kim HJ, et al. 
Simulation of the cardiovascular system using PID control model. J Korean Soc Med Inform 2004;10:367-377.

23. Benenson W, Harris JW, Stocker H, Lutz H. Handbook of physics. 1st ed. New York: Springer, 2002;501-543.

24. Shimizu Y, Itoh T, Hougaku H, Nagai Y, Hashimoto H, Sakaguchi $M$, et al. Clinical usefulness of duplex ultrasonography for the assessment of renal arteriosclerosis in essential hypertensive patients. Hypertens Res 2001;24:13-17.

25. Ohta Y, Fujii K, Arima H, Matsumura K, Tsuchihashi T, Tokumoto $M$, et al. Increased renal resistive index in atherosclerosis and diabetic nephropathy assessed by Doppler sonography. J Hypertens 2005;23:1905-1911.

26. Mostbeck GH, Gossinger HD, Mallek R, Siostrzonek P, Schneider $B$, Tscholakoff D. Effect of heart rate on Doppler measurements of resistive index in renal arteries. Radiology 1990;175:511-513.

27. Kublickas M, Randmaa I, Lunell NO, Westgren M. Effect of variations of heart rate within the normal range on renal artery Doppler indices in nonpregnant and pregnant women. J Clin Ultrasound 1993;21:507-510. 